\title{
New Advances on Fibroblast Growth Factor-Based Coatings for Hip Replacement Implants
}

\author{
Ionela Cristina Nica 1,*, Miruna S. Stan ${ }^{1,2,3}$, Anca Dinischiotu ${ }^{1}$, Valentina Grumezescu ${ }^{4}$, \\ Alexandra Elena Stoica ${ }^{2}$, Alina Holban ${ }^{3,5}$, Alexandru Mihai Grumezescu ${ }^{2}$ \\ 1 Department of Biochemistry and Molecular Biology, Faculty of Biology, University of Bucharest, 91-95 \\ Splaiul Independentei, 050095 Bucharest, Romania; cristinai.nica@gmail.com (I.C.N.); \\ miruna_stan@yahoo.com (M.S.S.); ancadinischiotu@yahoo.com (A.D.) \\ 2 Department of Science and Engineering of Oxide Materials and Nanomaterials, Faculty of Applied \\ Chemistry and Materials Science, University Politehnica of Bucharest, 1-7 Polizu Street, 011061 Bucharest, \\ Romania; miruna_stan@yahoo.com (M.S.S.); elena_oprea_93@yahoo.co.uk (A.E.S.); \\ grumezescu@yahoo.com (M.G.) \\ 3 Research Institute of the University of Bucharest -ICUB, 91-95 Splaiul Independentei, 050095 Bucharest, \\ Romania; miruna_stan@yahoo.com (M.S.S.); alina_m_h@yahoo.com (A.H.) \\ 4 National Institute for Lasers, Plasma and Radiation Physics, Măgurele 077125, Romania; \\ valentina.grumezescu@inflpr.ro (V.G.) \\ 5 Microbiology Immunology Department, Faculty of Biology, University of Bucharest, 1-3 Portocalelor Lane, \\ Sector 5, 77206 Bucharest, Romania; alina_m_h@yahoo.com (A.H.) \\ * Correspondence: cristinai.nica@gmail.com (I.C.N.)
}

Received: 11 March 2020; Accepted: 14 May 2020; Published: 15 May 2020

\begin{abstract}
It is already well known that the tissue-implant interface is one of the most critical factors for the success of the implant integration. The use of bioactive and biomimetic surfaces is of great interest in biomedical applications especially in tissue engineering. Therefore, in our study we aimed to obtain successful coatings based on hydroxyapatite, antibiotics and growth factors in order to increase the biocompatibility of commercial implant materials by promoting cell attachment and growth without toxic effects as well as inhibition of microbial biofilm formation. In this way, homogenous mixtures of hydroxyapatite, kanamycin and fibroblast growth factor (HAP/KAN, $\mathrm{HAP} / \mathrm{FGF}$ and HAP/KAN/FGF) were coated on titanium-based metal plates for hip replacement implants. The coatings were able to impair the initial adherence of bacterial cells and to reduce the biofilm formation throughout the release of antibiotic. The cytocompatibility of these samples was investigated on murine normal osteoblasts (MC3T3-E1 cell line) with fibroblast-like morphology by evaluating their influence on cellular viability and potential to generate an inflammatory response. In addition, the adhesion and proliferation, as well as the actin cytoskeleton organization, were observed after $24 \mathrm{~h}$ of cell culture on these coatings. The results confirmed the biocompatibility of all coatings, the cell number counted for HAP/KAN/FGF sample being equal to control. Since it is well known that NO is a marker of inflammation with an essential role in regulating apoptotic death and cell viability, our study showed that cell growth on these surfaces did not induce nitric oxide (NO) release, NO level being maintained close to control values for all tested samples. Also, an excellent cell adherence and spreading on these coatings deposited on hip implants was evidenced by fluorescence microscopy, supporting their usage as substrates in tissue engineering applications. Acknowledgements. This work has been funded by the Operational Programme Human Capital of the Ministry of European Funds through the Financial Agreement 51668/09.07.2019, SMIS code 124705, and through the project no. 77PD/2018 NANO-BIO-INT (PN-III-P1-1.1-PD-2016-1562).
\end{abstract}

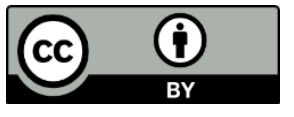

(C) 2020 by the authors. Submitted for possible open access publication under the terms and conditions of the Creative Commons Attribution (CC BY) license (http://creativecommons.org/licenses/by/4.0/). 